\title{
Odontohypophosphatasia treated with asfotase alfa enzyme replacement therapy in a toddler: a case report
}

\author{
Mizuki Takagi ${ }^{1}$, Shunsuke Kato ${ }^{2}$, Taichiro Muto ${ }^{3}$, Yoshimi Sano ${ }^{4}$, Tomoyuki Akiyama ${ }^{5}$, Junko Takagi ${ }^{6}$, \\ Akihisa Okumura ${ }^{3}$, and Hideyuki Iwayama ${ }^{3}$

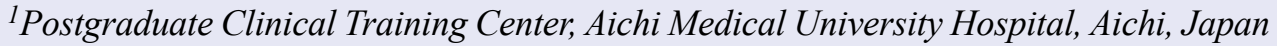 \\ ${ }^{2}$ Department of Pediatrics, Kasugai Municipal Hospital, Aichi, Japan \\ ${ }^{3}$ Department of Pediatrics, Aichi Medical University, School of Medicine, Aichi, Japan \\ ${ }^{4}$ Division of Pediatric Dentistry and Orthodontics, Department of Plastic and Reconstructive Surgery, Fujita Health \\ University Department of Plastic Surgery, Aichi, Japan \\ ${ }^{5}$ Department of Child Neurology, Okayama University Hospital, Okayama, Japan \\ ${ }^{6}$ Division of Endocrinology and Metabolism, Department of Internal Medicine, Aichi Medical University, Aichi, \\ Japan
}

\begin{abstract}
Hypophosphatasia (HPP) is a rare skeletal disorder caused by loss-of-function mutations in Alkaline Phosphatase, Biomineralization associated $(A L P L)$ gene that encodes tissue-nonspecific alkaline phosphatase. Odontohypophosphatasia (odonto-HPP), a mild form of HPP, is characterized only by oral manifestations including premature exfoliation of deciduous teeth. Enzyme replacement therapy (ERT) is effective in severe HPP cases; however, information about its efficacy for odonto-HPP is limited. A 2-yr-old girl was referred to our hospital for mobility of her deciduous teeth with low serum alkaline phosphatase (ALP) level of $253 \mathrm{U} / \mathrm{L}$ (reference range: 410-1,150 U/L) and high urine phosphoethanolamine level of $1,419.9 \mu \mathrm{mol} / \mathrm{g} \cdot$ Cre $(7-70 \mu \mathrm{mol} / \mathrm{g} \cdot \mathrm{Cre})$. She had no history of bone fractures; however, several members of her family had low serum ALP levels with a history of pathological fractures. She had a novel heterozygous missense mutation (c.1183A>T, p.Ile395Phe) in ALPL, and therefore, was diagnosed with odonto-HPP. After she was provided ERT to prevent premature exfoliation, no tooth mobility was observed. However, two deciduous teeth exfoliated two months after starting ERT, which was possibly triggered by a bout of common cold. Starting ERT following tooth mobility might be relatively late. Previous studies on experimental mice showed that starting ERT at birth may be effective in preventing premature exfoliation of deciduous teeth.
\end{abstract}

Key words: odontohypophosphatasia, premature exfoliation, deciduous teeth, enzyme replacement therapy, asfotase alfa

\section{Introduction}

Hypophosphatasia (HPP) is an inherited systemic skeletal disease caused by loss-of-function mutations in Alkaline Phosphatase, Biomineralization associated $(A L P L)$ gene that encodes tissue-nonspecific alkaline phosphatase (TNSALP) (1). Deficient TNSALP activity leads to accumulation of inorganic pyrophosphate, which is the substrate of alkaline phosphatase (ALP) and an inhibitor of hydroxyapatite formation (2), resulting in defective bone and/or teeth mineralization (3). ALP dysfunction in the teeth induces demineralization of the enamel, dentin, cementum, and alveolar bone.

HPP is classified into six forms according to the age of onset and severity: perinatal lethal, perinatal benign, infantile, childhood, adult, and odontohypophosphatasia (odonto-HPP) (3). Perinatal and most cases of infantile HPP are inherited in an autosomal recessive manner. The milder forms, such as childhood HPP, adult HPP, and odonto-HPP, are inherited in an autosomal recessive or autosomal dominant manner with the TNSALP activity affected by the $A L P L$ pathogenic variant (3). In Japan, the birth prevalence of severe HPP is reportedly 1:150,000 (4); however, the prevalence of its milder forms

Received: February 26, 2020 Accepted: March 16, 2020

Corresponding Author: Hideyuki Iwayama, M.D., Ph.D., Department of Pediatrics, Aichi Medical University, School of Medicine, 1-1 Yazakokarimata, Nagakute, Aichi 480-1195, Japan

E-mail: iwahide1976@gmail.com 
-such as odonto-HPP- remains unknown.

Enzyme replacement therapy (ERT) using bonetargeted recombinant ALP or asfotase alfa (Strensiq ${ }^{\mathrm{TM}}$; ALEXION, Tokyo, Japan) has been reported to be effective against severe HPP. In severe HPP, such as perinatal and infantile HPP, progressive chest deformity leads to an insufficiency of the respiratory system, and the survival rate is reported to be $42 \%$ and $27 \%$ at the ages of 1 and $5 \mathrm{yr}$, respectively (5). Asfotase alfa improves the mineralization of the bone, including the ribs, and respiratory function, and is mostly used in cases of severe HPP (5-7).

Only one case of odonto-HPP treated with asfotase alfa was reported (8); however, its clinical course and the effectiveness of asfotase alfa against odonto-HPP has not been reported. This is the first study to have reported the effectiveness of ERT in odonto-HPP in a 2-yr- and 2-mo-old girl.

\section{Case Report}

At the age of $1 \mathrm{yr}$, the patient was incidentally found to have low serum ALP levels during testing for food allergy. At the age of $2 \mathrm{yr}$ and $2 \mathrm{mo}$, she showed mobility of her deciduous teeth (Miller's Mobility Index Grade 3) and was referred to our hospital for further examination. The patient was born following full-term pregnancy with a birth weight of $3,374 \mathrm{~g}$, body length of $48.5 \mathrm{~cm}$, head circumference of $33 \mathrm{~cm}$, and chest circumference of 31 $\mathrm{cm}$ (all within normal ranges). She had no significant medical history related to bone abnormalities, fractures, convulsions, or delay in walking and speaking. On examination, her body weight and height were $11.0 \mathrm{~kg}$ $(-0.33 \mathrm{SD})$ and $85.0 \mathrm{~cm}(-0.25 \mathrm{SD})$, respectively. She had no gait abnormality. Oral examination revealed mobility of both deciduous mandibular central incisors and one lateral incisor with deep pockets $(4 \mathrm{~mm}$ ) (normal range: $1-3 \mathrm{~mm}$ ).

Her family history revealed bone fractures in her parents, paternal aunt, and paternal grandfather as well as low serum ALP levels in her parents and paternal aunt (Fig. 1). Her father had avulsion fractures three times, and her paternal aunt had a humeral fracture during their childhood. None of her relatives had dental abnormalities.

Laboratory evaluation revealed a low serum ALP level of $253 \mathrm{U} / \mathrm{L}$ (reference range, 410-1,150 U/L, measured via the method of Japan Society of Clinical Chemistry). The levels of the isozymes ALP2 and ALP3 were 43 U/L (reference range, 42-148 U/L) and 210 U/L (reference range, 43-151 U/L), respectively. Elevated levels of phosphoethanolamine (PEA), a substrate of ALP, were observed in her urine $(1,419.9$ $\mu \mathrm{mol} / \mathrm{g}$.Cre; reference range, $7-70 \mu \mathrm{mol} / \mathrm{g}$ Cre). Serum calcium $(9.7 \mathrm{mg} / \mathrm{dL}$; reference range, $8.7-10.3 \mathrm{mg} / \mathrm{dL})$, phosphorus ( $5.8 \mathrm{mg} / \mathrm{dL}$; reference range, $3.5-5.9 \mathrm{mg} / \mathrm{dL})$, magnesium ( $1.9 \mathrm{mg} / \mathrm{dL}$; reference range, $1.7-2.3 \mathrm{mg} /$ $\mathrm{dL}$ ), and intact PTH (42 pg/dL; reference range, 10-65 $\mathrm{pg} / \mathrm{dL}$ ) levels were within normal ranges. Dental X-ray

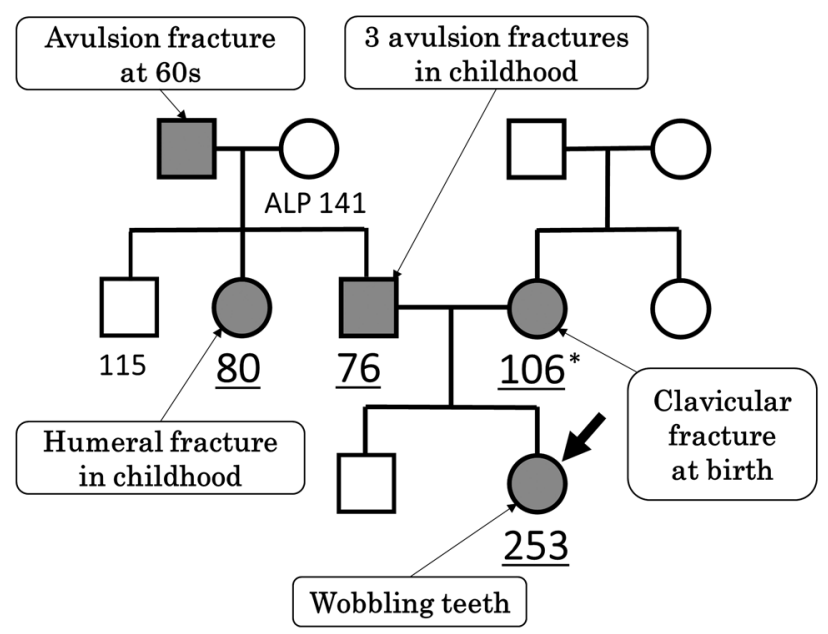

Fig. 1. Pedigree of the patient. The arrow indicates the proband. The normal range of alkaline phosphatase (ALP) for adults is 115-359 (U/L). Underlining of a number indicates that the ALP is decreased. Asterisk $(*)$ indicates that there was a change in serum ALP during the perinatal period; 150 during lactation for the first child; 98 during pregnancy for the second child (this case); 106 at the end of the lactation for the second child.
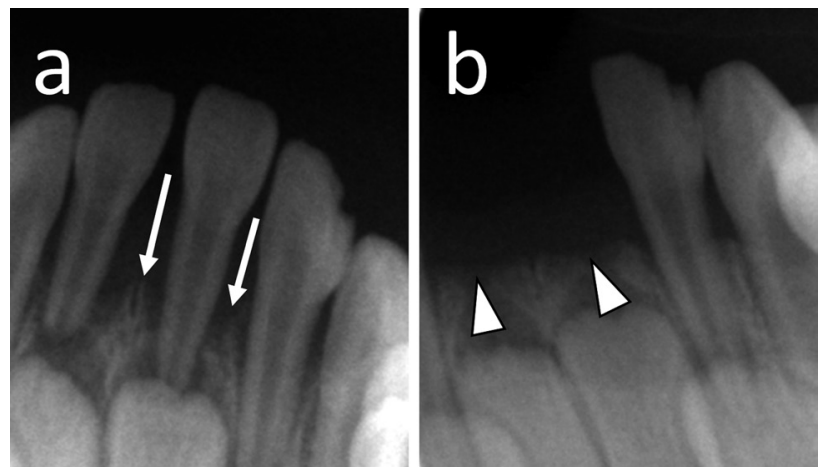

Fig. 2. Dental X-ray showed alveolar bone absorption in the regions of the deciduous mandibular central incisors. The arrows indicate the absorption of the alveolar bone, whereas the arrow shafts indicate the normal thickness of the alveolar bone (a). The arrowheads indicate the exfoliation of the deciduous mandibular incisors (b). a: before the exfoliation; b: after the exfoliation.

showed alveolar bone resorption in the regions of the deciduous mandibular left central incisors (Fig. 2a). No characteristics related to rickets or osteomalacia were observed on the X-ray of the trunk as well as the upper and lower extremities. Genetic testing showed a novel heterozygous missense mutation (c.1183A $>\mathrm{T}$, p.Ile395Phe) in $A L P L$.

Her mother had a clavicular fracture at birth; however, no other fractures were observed later on and because no bone fractures were reported in the proband's maternal family line, her mother did not undergo genetic 

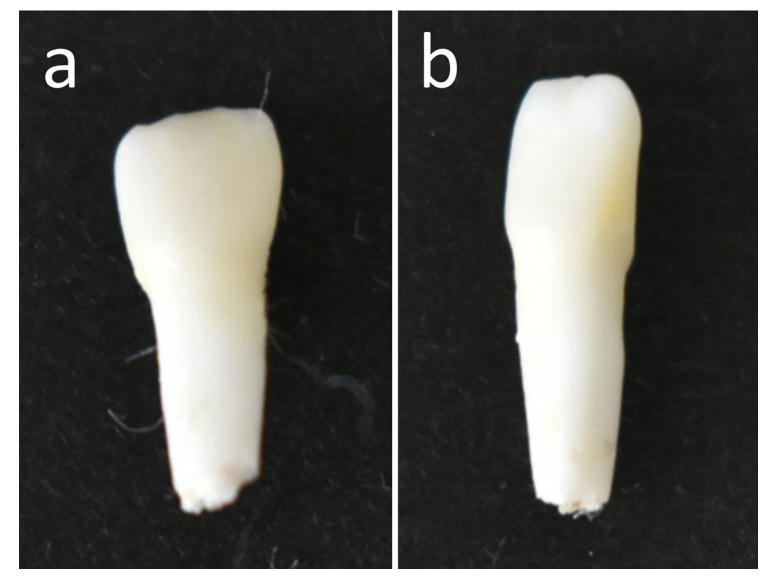

Fig. 3. The exfoliated teeth with the root. a: right deciduous mandibular central incisor; b: left deciduous mandibular central incisor.

testing. Moreover, her father rejected undergoing genetic testing. Her paternal uncle had the same mutation in $A L P L$ as that of the proband, whereas her paternal grandfather had no mutation. Her paternal grandmother did not visit our hospital.

Odonto-HPP was diagnosed based on the history of mobility of deciduous teeth with a marked decrease in serum ALP and elevated urine PEA levels, family history, and mutation in ALPL. After diagnosis of odonto-HPP, the patient received ERT with subcutaneous injections of $2 \mathrm{mg} / \mathrm{kg}$ asfotase alfa thrice a week. One month after starting ERT, her serum ALP level increased to 23,280 U/L. She began to put her hand in her mouth and bite a towel; such behavior was not seen before the treatment. No new mobile teeth were noticed after starting ERT. However, exfoliation of two deciduous teeth that were already mobile was triggered by catching a cold 2 mo after starting ERT (Fig. 2b). The roots of the teeth remained attached to the lost teeth (Fig. 3a, 3b), which is often seen in HPP (3). To date, she has been receiving ERT at home without any adverse effects, bone fractures, or developmental delay.

This study was approved by the Ethical Committee at the Aichi Medical University (2019-145). All evaluations and procedures were performed in accordance with the tenets of the Declaration of Helsinki. We collected clinical data retrospectively during routine medical practice. Informed consent for publication was obtained from the patient's parents.

Discussion

\section{Why do deciduous teeth exfoliate in HPP?}

In HPP, premature exfoliation of teeth is considered to be caused by defective development of acellular cementum, which is the tissue that anchors a tooth to the alveolar bone via the alveolar ligament (9). Therefore, exfoliated teeth have intact roots, which are often seen in HPP (3). In the present case, the two exfoliated teeth had morphologically normal roots. Thus, it is reasonable to consider that tooth exfoliation occurred because of the weakness in the connection between the cementum and the alveolar bone and not owing to the dissolution of the tooth roots. Premature exfoliation of deciduous teeth also causes alveolar bone defects, leading to misalignment or premature exfoliation of permanent teeth (3). To avoid these abnormalities of the alveolar bone and the permanent teeth, it is important to prevent premature exfoliation of deciduous teeth.

\section{Is ERT effective in treating odonto-HPP?}

To the best of our knowledge, this is the first study that described a case of odonto-HPP treated with ERT. No new mobile teeth were noticed after ERT started. However, exfoliation of two deciduous teeth that were already mobile was triggered by catching a cold 2 mo after starting ERT. Millán et al. reported that ERT was effective on the teeth of $A l p l^{-/-}$mice aged $16 \mathrm{~d}$ (10). Wildtype (WT) mice showed complete dentin mineralization. $\mathrm{Alpl}^{-1-}$ mice showed only partial mineralization of dentin and the alveolar bone, whereas ERT-treated $\mathrm{Alpl}^{-1-}$ mice showed complete mineralization of these structures such that no differences in mineralization of incisors were seen between ERT-treated $A l p l^{-1-}$ mice and WT mice. In the present case, ERT was administered to the patient after deciduous teeth were already mobile, and premature exfoliation of the teeth could not be prevented. A possible reason for this could be that enamel, dentin, and cementum do not remodel after eruption. Therefore, starting ERT during the neonatal period is crucial when odontogenesis is complete.

Odontogenesis of deciduous teeth begins in the embryonic period between the $6^{\text {th }}$ and the $7^{\text {th }}$ wk of prenatal development (11). Between the $12^{\text {th }}$ and $14^{\text {th }}$ wk of prenatal development, the enamel, dentin, and acellular cementum begin to be produced by odontogenic cellular byproducts (11). Cellular cementum, another type of cementum, develops after most processes of odontogenesis are complete and after the tooth occludes with the tooth in the opposite arch (12). Therefore, it may be effective to start ERT from the $6^{\text {th }}$ week of prenatal development and $1.5 \mathrm{yr}$ after birth when cellular cementum is completed (13).

In odonto-HPP, the mean number of deciduous teeth that exfoliate prematurely is 4.2 (14). In the present case, the current number of lost teeth is 2 and none of her remaining teeth are mobile. If she has no more exfoliated teeth until she turns $4 \mathrm{yr}$ old, it may be fair to say that ERT is effective in odonto-HPP to prevent early exfoliation of deciduous teeth.

\section{Will the diagnosis of odonto-HPP be altered to chilldhood HPP?}

In the present case, the patient showed only oral manifestations based on which the diagnosis of odontoHPP was made; however, there remains a possibility of 
the diagnosis being changed to childhood HPP. Mori et al. reported that 7 out of 9 cases diagnosed with HPP showed premature exfoliation of deciduous teeth; their median age was 14 yr (range, 6-19 yr) when they had their first bone fractures (15). Moreover, the number of prematurely exfoliated deciduous teeth correlated with HPP severity (14). In the present case, the patient had tooth exfoliation at the age of $2 \mathrm{yr}$ and $4 \mathrm{mo}$, and the diagnosis of odonto-HPP may be reserved until much later in her life when no extraoral manifestations are evident after long-term follow-up (14).

\section{Conclusion}

We could not prevent the exfoliation of deciduous teeth despite administering ERT in a patient with odonto-HPP. Starting ERT at a younger age may be necessary to avoid exfoliation of deciduous teeth.

Conflict of Interests: The authors have no conflicts of interest relevant to this article to disclose.

\section{Acknowledgements}

We would like to express our gratitude to the staff of Aichi Medical University.

\section{References}

1. Bowden SA, Foster BL. Profile of asfotase alfa in the treatment of hypophosphatasia: design, development, and place in therapy. Drug Des Devel Ther 2018;12: 3147-61. [Medline] [CrossRef]

2. Addison WN, Azari F, Sørensen ES, Kaartinen MT, McKee MD. Pyrophosphate inhibits mineralization of osteoblast cultures by binding to mineral, up-regulating osteopontin, and inhibiting alkaline phosphatase activity. J Biol Chem 2007;282: 15872-83. [Medline] [CrossRef]

3. Mornet E, Nunes ME. Hypophosphatasia. In: Adam MP, Ardinger HH, Pagon RA, Wallace SE, Bean LJH, Stephens K, et al., editors. GeneReviews(R). Seattle (WA): University of Washington, Seattle. GeneReviews is a registered trademark of the University of Washington, Seattle. 1993.

4. Michigami T, Uchihashi T, Suzuki A, Tachikawa K, Nakajima S, Ozono K. Common mutations F310L and T1559del in the tissue-nonspecific alkaline phosphatase gene are related to distinct phenotypes in Japanese patients with hypophosphatasia. Eur J Pediatr 2005;164: 277-82. [Medline] [CrossRef]

5. Whyte MP, Rockman-Greenberg C, Ozono K, Riese R, Moseley S, Melian A, et al. Asfotase alfa treatment improves survival for perinatal and infantile hypophosphatasia. J Clin Endocrinol Metab 2016;101: 334-42. [Medline] [CrossRef]

6. Whyte MP, Greenberg CR, Salman NJ, Bober MB, McAlister WH, Wenkert D, et al. Enzyme-replacement therapy in life-threatening hypophosphatasia. N Engl J Med 2012;366: 904-13. [Medline] [CrossRef]

7. Kitaoka T, Tajima T, Nagasaki K, Kikuchi T, Yamamoto K, Michigami T, et al. Safety and efficacy of treatment with asfotase alfa in patients with hypophosphatasia: Results from a Japanese clinical trial. Clin Endocrinol (Oxf) 2017;87: 10-9. [Medline] [CrossRef]

8. Okawa R, Kokomoto K, Kitaoka T, Kubota T, Watanabe A, Taketani T, et al. Japanese nationwide survey of hypophosphatasia reveals prominent differences in genetic and dental findings between odonto and non-odonto types. PLoS One 2019;14: e0222931. [Medline] [CrossRef]

9. Bloch-Zupan A, Vaysse F. Hypophosphatasia: oral cavity and dental disorders. Arch Pediatr 2017;24(5S2): S80, S84. [Medline] [CrossRef]

10. Millán JL, Narisawa S, Lemire I, Loisel TP, Boileau G, Leonard P, et al. Enzyme replacement therapy for murine hypophosphatasia. J Bone Miner Res 2008;23: 777-87. [Medline] [CrossRef]

11. Adserias-Garriga J, Visnapuu V. The neonatal line as evidence of live birth. In: Adserias-Garriga J, editor. Age estimation a multidisciplinary approach. Histological approach of age estimation. 3. San Marcos, TX, USA: Academic press; 2019. p.161-68.

12. Premkumar S. Development of human dentition, supporting structures and occlusion. In: Premkumar S, editor. Text book of craniofacial growth. New Delhe, India: Jaypee brothers medical publishers (P) Ltd; 2011. p.109-36.

13. Ash M, Nelson S. Dental anatomy, physiology, and occulusion. In: Ash M, editor. Wheeler's dental anatomy, physiology, and occlusion. 8 ed. Philadelphia: Saunders; 2003. p.29-64.

14. Whyte MP, Zhang F, Wenkert D, McAlister WH, Mack KE, Benigno MC, et al. Hypophosphatasia: validation and expansion of the clinical nosology for children from 25 years experience with 173 pediatric patients. Bone 2015;75: 229-39. [Medline] [CrossRef]

15. Mori M, DeArmey SL, Weber TJ, Kishnani PS. Case series: Odontohypophosphatasia or missed diagnosis of childhood/ adult-onset hypophosphatasia? - Call for a long-term follow-up of premature loss of primary teeth. Bonekey Rep 2016;5: 228-32. [Medline] [CrossRef] 